\title{
Conocimiento y cumplimiento de medidas de bioseguridad en personal de enfermería. Hospital Nacional Almanzor Aguinaga. Chiclayo 2002
}

\author{
VÍCTOR SOTO ${ }^{1}$, ENRIQUE OLANO ${ }^{2}$ \\ ${ }^{1}$ Jefe Oficina Epidemiología y Programas Hospital Nacional Almanzor Aguinaga. \\ ${ }^{2}$ Coordinador Centro de Prevención de Riesgos del Trabajo.
}

\begin{abstract}
Resumen
Objetivo: Determinar el nivel de conocimientos y cumplimiento de las medidas de bioseguridad del personal profesional y técnico de enfermería que labora en áreas de alto riesgo. Material y Métodos: Estudio transversal, descriptivo, siendo la población el personal profesional y técnico de enfermería que laboraba en Emergencia, Unidad de Cuidados Intensivos (UCI), Unidad de Cuidados Intermedios (UCEMIN), Cirugía General, Centro Quirúrgico, Neonatología y Hemodiálisis del Hospital Nacional Almanzor Aguinaga EsSalud de Chiclayo; se tomó una muestra de 117 trabajadores, evaluándose el nivel de conocimientos mediante cuestionario y el cumplimiento mediante una guía de observación. Resultados: UCI y Centro Quirúrgico tuvieron un nivel de conocimientos alto, tanto en profesionales como técnicos de enfermería. Sobre cumplimiento de normas de bioseguridad, los resultados fueron variados, siendo el mayor en centro quirúrgico y el menor en cirugía y UCEMIN. Existen errores comunes: mal uso de guantes no realizando cambio oportuno, menor frecuencia de lavado de manos, consumo de alimentos en áreas no adecuadas, reencapuchado de las agujas, etc. Conclusiones: Existe un alto grado de conocimiento de las normas de bioseguridad por el personal profesional y técnico de enfermería; sin embargo, el cumplimiento de las normas de bioseguridad es en promedio de nivel 2 (30 a 60\%).
\end{abstract}

Palabras clave: Medidas de seguridad; servicio de urgencia en hospital; cuidados intensivos; cuidados críticos.

Knowledge and fulfillment of biosecurity standards in nurse personal. Almanzor Aguinaga National Hospital. Chiclayo 2002

\section{Abstract}

Objectives: To determine knowledge and fulfilment of biosecurity standards in nurses and nurse technicians who work in high risk areas. Material and Methods: Transversal, prospective and descriptive study. Population included nurses and nurse technicians who work at Emergency, Intensive Care Unit (ICU), Intermediate Care Unit (UCEMIN), General Surgery, Surgical Center, Neonatology and Hemodialysis services at Chiclayo Almanzor Aguinaga National Hospital, EsSalud. The sample included 117 workers, sample obtained by systematic and probabilistic selection. Knowledge level was determined by questionnaire and fulfilment by observation guide levels. Results: ICU and Surgical Center's nurses and nurse technicians had a high level of knowledge in biosecurity standards; fulfilment varied from high fulfilment at the Surgical Center and least at General Surgery and UCEMIN; there were several common mistakes, such as, bad use of gloves, low frequency of hand washing, eating foods in not allowed places and needle hooding. Conclusions: There is a high level of knowledge on biosecurity standards in nurses and nurse technicians but fulfilment of the standards was level 2 (30 to 60\%) only.

Key words: Security measures; emergency service, hospital; intensive care; critical care.

Correspondencia:

Dr. Víctor Soto Cáceres

Francisco Cabrera 240. Chiclayo, Perú.

E-mail: victor.soto@essalud.gob.pe vicsoca@hotmail.com 


\section{INTRODUCCIÓN}

En el Seguro Social (EsSalud) se dispone de normas de bioseguridad que están destinadas a reducir el riesgo de transmisión de microorganismos de fuentes reconocidas o no reconocidas de infección, vinculadas a accidentes por exposición a sangre y fluidos corporales. En el año 2001, se difundió a todos los servicios médicos una nueva Directiva sobre prácticas de bioseguridad $\left({ }^{1}\right)$, bajo estos principios:

A) Universalidad: Las medidas deben involucrar a todos los pacientes de todos los servicios, independientemente de conocer o no su serología. Todo el personal debe seguir las precauciones estándares rutinariamente para prevenir la exposición de la piel y de las membranas mucosas, en todas las situaciones que puedan dar origen a accidentes. Estas precauciones, deben ser aplicadas para TODAS las personas, independientemente de presentar o no patologías $(2,3)$.

B) Uso de barreras: Comprende el concepto de evitar la exposición directa a sangre y otros fluidos orgánicos potencialmente contaminantes, mediante la utilización de materiales adecuados que se interpongan al contacto de los mismos. La utilización de barreras (ej. guantes) no evitan los accidentes de exposición a estos fluidos, pero disminuyen las consecuencias de dicho accidentes.

C) Medios de eliminación de material contaminado: Comprende el conjunto de dispositivos y procedimientos adecuados a través de los cuales los materiales utilizados en la atención de pacientes, son depositados y eliminados sin riesgo $\left.{ }^{4-6}\right)$.

Según la literatura, 65 a $70 \%$ de los accidentes ocurren en el personal de enfermería, seguido del personal de limpieza $(17 \%)$, luego el personal de laboratorio (10 a $15 \%$ ) y finalmente el personal médico $(4 \%)\left({ }^{7}\right)$.

Los accidentes ocurren con más frecuencia en la habitación del enfermo (60 a 70\%) y en una Unidad de Cuidados Intensivos (10 a 15\%) $\left.{ }^{2}\right)$. Dentro del personal de enfermería, las circunstancias en que ocurren las punciones son principalmente al administrar medicamentos $(30 \%)$, seguido de la práctica de reencapuchar la aguja $(24 \%)\left({ }^{8}\right)$.

Una monografía que compara una institución pública de salud con otra privada, en la Argentina, respecto a accidentes cortopunzantes con material patogénico, encontró accidentes en enfermería en igualdad de casos absolutos, con mayor tasa en sector privado por la población de trabajadores; siendo lo contrario al comparar tasa de accidentes en médicos residentes del sector público $(3 \%)$ con el privado $(1 \%)$; sin embargo, lo más llamativo fue con el personal de limpieza y recolectores de residuos, con tasa de $58 \%$ en institución pública (servicio tercerizado) y tan sólo $10 \%$ en institución privada; esto indica que no se está implementando las medidas de bioseguridad y proporcionando los implementos necesarios de protección a los trabajadores $\left({ }^{9}\right)$.

Un estudio entre las clínicas odontológicas de la región metropolitana de Santiago de Chile demostró que $74,3 \%$ del equipo odontológico tiene conocimiento del Manual de Normas y 79\% consideró muy necesario su aplicación; sin embargo, un tercio del equipo declaró haber tenido algún accidente laboral en los últimos seis meses $\left({ }^{10}\right)$.

En otros grupos ocupacionales, como los de funerarias, que generan residuos peligrosos similares a los hospitalarios denominados tanatopráxicos, se investigó al personal expuesto en laboratorios de nueve funerarias de Medellín (Colombia), encontrándose que $98 \%$ conocían los factores de riesgo ocupacionales; sin embargo, $60 \%$ de ellos no se protegía adecuadamente. Por otro lado, los esquemas de vacunación contra hepatitis B estaban incompletos y un $43 \%$ no tenía un nivel adecuado de seroconversión para su oficio $\left({ }^{11}\right)$.

En el Perú, se encuentra algunos trabajos relacionados con conocimientos de bioseguridad en relación al VIH/SIDA; y, en Juliaca, 
compararon el nivel de conocimiento y actitudes de los médicos y paramédicos de los hospitales Carlos Monge Medrano y el Hospital IPSS de Juliaca, en 1997, encontrando buen nivel de conocimientos en ambos lugares y en los distintos grupos ocupacionales. Concluyen con que los conocimientos están relacionados con las actitudes en ambos hospitales $\left({ }^{12}\right)$.

Un estudio realizado en el año 2000 en el Hospital Nacional Almanzor Aguinaga Asenjo (HNAAA) con estudiantes de medicina, aplicando un cuestionario a una muestra de trabajadores asistenciales en forma estratificada, encontró que $24 \%$ tenía deficiente conocimiento de las medidas de bioseguridad, siendo mayor este déficit en obstetrices y técnicos de enfermería; sólo $40 \%$ de los trabajadores refería haber recibido charlas de bioseguridad, a pesar de la información oficial de que el CEPRIT (Centro de prevención de riesgos del trabajo) institucional había completado capacitaciones al respecto; el cumplimiento de las normas de bioseguridad era ocasional para $67 \%$, siendo mayor en internos de medicina, técnicos de enfermería y laboratorio $\left({ }^{11}\right)$.

Según los resultados del año 2000, era necesario verificar si todos los trabajadores del seguro social tenían un adecuado conocimiento de las medidas y normas de bioseguridad y en especial de su cumplimiento, que sirven tanto de protección para él y su familia como para el paciente a su cuidado, al haberse difundido oficialmente las normas en todos los servicios y haberse realizado diversas acciones de capacitación. Se consideró como grupo prioritario al personal de enfermería y técnicos de enfermería, por ser los que reportaban mayor número de accidentes por agujas durante su labor, lo que puede acarrear problemas legales de tipo laboral a la institución.

Nos planteamos verificar cuál era el nivel de conocimiento y cumplimiento de las medidas de bioseguridad del personal profesional y técnico de enfermería que labora en áreas de alto riesgo del HNAAA.

\section{MATERIAL Y MÉTODOS}

La investigación realizada es un estudio descriptivo de tipo transversal, siendo la población objeto de estudio el personal profesional y técnico de enfermería que labora en áreas de alto riesgo del HNAAA, las que se incluye: Emergencia, Unidad de Cuidados Intensivos, UCEMIN, Servicio de Cirugía general, Centro quirúrgico, Neonatología y Centro de hemodiálisis.

Para la estimación de la muestra, se aplicó la fórmula para obtener tamaño de muestra

$$
\mathrm{n}=\frac{\mathrm{Z}^{2} \mathrm{pq} \mathrm{N}}{\mathrm{Z}^{2} \mathrm{pq}+\mathrm{T}^{2}(\mathrm{~N}-1)}
$$

En la cual se consideró para los efectos del estudio los siguientes valores :

$$
\begin{aligned}
& Z=95 \%=0,95=1,94 \\
& p=60 \%=0,6 \quad q=40 \%=0,4 \\
& T=5 \%=0,05 \quad N=196
\end{aligned}
$$

La estimación dio como resultado una muestra de 117 trabajadores, los cuales fueron seleccionados según estratos con una secuencia de selección sistemática de inicio aleatorio, entre el personal de enfermería de los servicios de alto riesgo.

Para efectos del estudio se estableció las siguientes definiciones operacionales:

- Bioseguridad: Término empleado para reunir y definir las normas relacionadas con el comportamiento preventivo del personal del hospital frente a riesgos propios de su actividad diaria.

- Área de alto riesgo: Zona o espacio del centro asistencial de salud en donde por la naturaleza de sus actividades y procedimientos en la atención directa al usuario y manipulación de materiales, insumos y otros potencialmente contaminados con fluidos corporales, existe un mayor riesgo de adquirir infecciones en usuarios y trabajadores. 
- Procedimiento de alto riesgo: Es el procedimiento en la atención directa al usuario y manipulación de materiales, insumos y otros potencialmente contaminados con fluidos corporales, en el que existe un mayor riesgo de adquirir infecciones.

- Residuos biocontaminados (categoría A): son los contaminados con agentes patógenos que pueden tener altas concentraciones de microorganismos potencialmente peligrosos para quienes entran en contacto con ellos (color de bolsa roja).

- Residuo especial (categoría B): los que por sus características físicas y químicas pueden ser corrosivos, inflamables, tóxicos, explosivos, radioactivos y reactivos. (Color de bolsa amarilla)

- Residuos comunes (categoría C): no son peligrosos y debería considerarse a nivel de los residuos domésticos; incluye los generados en oficinas, proveniente de la preparación de alimentos y todo lo no caiga en las categorías A y B (color de bolsa negra).

Con relación a la técnica e instrumentos de recolección de datos:

- Se determinó el nivel de conocimiento sobre medidas de bioseguridad que tiene el personal profesional y técnico de enfermería con cuestionario de conocimientos; algunas preguntas fueron tomadas del Protocolo del MINSA $\left({ }^{12}\right)$.

- Se evaluó el nivel de cumplimento de normas por profesionales y técnicos de enfermería mediante la observación realizada por el personal investigador, utilizando como instrumento una guía de observación de prácticas basada en el protocolo del MINSA.

Se identificó mediante observación rigurosa el o los servicios con mayores deficiencias en el cumplimiento de normas de bioseguridad.

En el cuestionario de conocimientos se calificó cada ítem correcto 2 puntos, excepto en la pregunta 6 , en la que el orden correcto vale 2 puntos, incorrecto 0 puntos.

\section{Calificación:}

- Conocimientos de bioseguridad

< 22 puntos: conocimiento bajo

22 a 44 puntos: conocimiento regular

$>44$ puntos conocimiento alto

La guía de observaciones prácticas fue calificada de la siguiente manera:

- Calificación dependiente del personal:

0 - 8 puntos Nivel 1 (<30\% de cumplimiento)

9 -16 puntos Nivel 2 (30 a $60 \%$ de cumplimiento)

$>16$ puntos Nivel 3 (>60\% de cumplimiento)

El análisis de la información se hizo mediante estadística descriptiva, aplicándose la prueba de chi cuadrado cuando se deseaba establecer asociación entre variables.

\section{RESULTADOS}

La distribución de la población y la obtención de la muestra por servicio determinó que el Centro Quirúrgico sea el área de mayor población, con $29,1 \%$ del total, seguido de Emergencia 19,9\% (Tabla 1).

Se obtuvo que $100 \%$ del personal del servicio de UCI y Centro quirúrgico tienen un grado de conocimiento alto acerca de normas de bioseguridad. En los servicios restantes, el grado

Tabla 1.- Distribución de la población y muestra de enfermeras y técnicas de enfermería según servicio.

\begin{tabular}{lccc}
\hline \multicolumn{1}{c}{ Servicio } & Población & Porcentaje & Muestra \\
\hline UCI & 17 & 8,7 & 10 \\
UCEMIN & 22 & 11,2 & 13 \\
Cirugía & 18 & 9,2 & 11 \\
C.Quirúrgico & 57 & 29,1 & 34 \\
Neonatología & 21 & 10,7 & 13 \\
Emergencia & 39 & 19,9 & 23 \\
Hemodiálisis & 22 & 11,2 & 13 \\
Total & 196 & 100,0 & 117 \\
\hline
\end{tabular}


de conocimiento se encontró entre los parámetros alto y regular; no hubo uno con bajo conocimiento (Tabla 2). En la Figura 1 se aprecia que $85,5 \%$ del personal tiene un buen nivel de conocimiento y el $14,5 \%$ un regular nivel.

Tabla 2.- Grado de conocimiento de normas de bioseguridad según personal profesional y técnico de enfermería.

\begin{tabular}{|c|c|c|c|c|}
\hline \multirow[t]{3}{*}{ Servicio } & \multicolumn{4}{|c|}{$\begin{array}{c}\text { Grado de conocimiento sobre } \\
\text { bioseguridad }\end{array}$} \\
\hline & \multicolumn{2}{|c|}{ Alto } & \multicolumn{2}{|c|}{ Regular } \\
\hline & $\mathrm{n}$ & $\%$ & $\mathrm{n}$ & $\%$ \\
\hline UCI & 10 & 100,0 & 0 & 0 \\
\hline UCEMIN & 7 & 53,8 & 6 & 46,2 \\
\hline Cirugía & 8 & 72,7 & 3 & 27,3 \\
\hline C.Quirúrgico & 34 & 100 & 0 & 0,0 \\
\hline Neonatología & 11 & 84,6 & 2 & 15,4 \\
\hline Emergencia & 20 & 87 & 3 & 13 \\
\hline Hemodiálisis & 10 & 76,9 & 3 & 23,1 \\
\hline Total & 100 & 85,5 & 17 & 14,5 \\
\hline
\end{tabular}

En el nivel de cumplimiento de normas de bioseguridad se observó lo siguiente (Tabla 3):

- E1 92\% del personal del servicio de Hemodiálisis tiene un cumplimiento nivel 3, seguido del Centro quirúrgico, UCI y

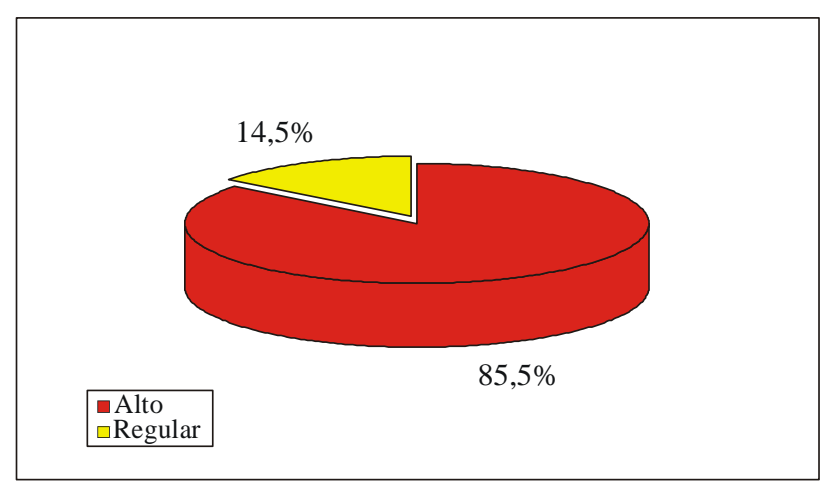

Figura 1.- Grado de conocimiento sobre bioseguridad. Profesionales y técnicas de enfermería. HNAAA 2002.
Tabla 3.- Nivel de cumplimiento de normas de bioseguridad.

\begin{tabular}{|c|c|c|c|c|c|c|}
\hline \multirow[t]{3}{*}{ Servicio } & \multicolumn{6}{|c|}{$\begin{array}{l}\text { Nivel de cumplimiento de } \\
\text { bioseguridad }\end{array}$} \\
\hline & \multicolumn{2}{|c|}{ Nivel 1} & \multicolumn{2}{|c|}{ Nivel 2} & \multicolumn{2}{|c|}{ Nivel 3} \\
\hline & $\mathrm{n}$ & $\%$ & $\mathrm{n}$ & $\%$ & $\mathrm{n}$ & $\%$ \\
\hline UCI & 0 & 0,0 & 2 & 20,0 & 8 & 80,0 \\
\hline UCEMIN & 5 & 38,5 & 8 & 61,5 & 0 & 0,0 \\
\hline Cirugía & 3 & 27,2 & 8 & 72,7 & 0 & 0,0 \\
\hline C.Quirúrgico & 0 & 0,0 & 4 & 11,8 & 30 & 88,2 \\
\hline Neonatología & 0 & 0,0 & 4 & 30,8 & 9 & 69,2 \\
\hline Emergencia & 1 & 4,4 & 15 & 65,2 & 7 & 30,4 \\
\hline Hemodiálisis & 1 & 7,7 & 0 & 0,0 & 12 & 92,3 \\
\hline Total & 10 & 8,5 & 41 & 35,0 & 66 & 56,5 \\
\hline
\end{tabular}

Neonatología $(88,2 ; 80$ y $69,2 \%$, respectivamente).

- El personal de Servicio de Cirugía, Emergencia y UCEMIN tienen un cumplimiento principalmente de nivel 2 $(72,7 ; 65,2$ y $61,5 \%)$. El nivel 1 de cumplimiento tiene una proporción de $40 \%$ solo en UCEMIN.

- En general, el nivel de cumplimiento es de nivel $356,5 \%$, nivel II $35 \%$ y nivel $18,5 \%$ (Figura 2).

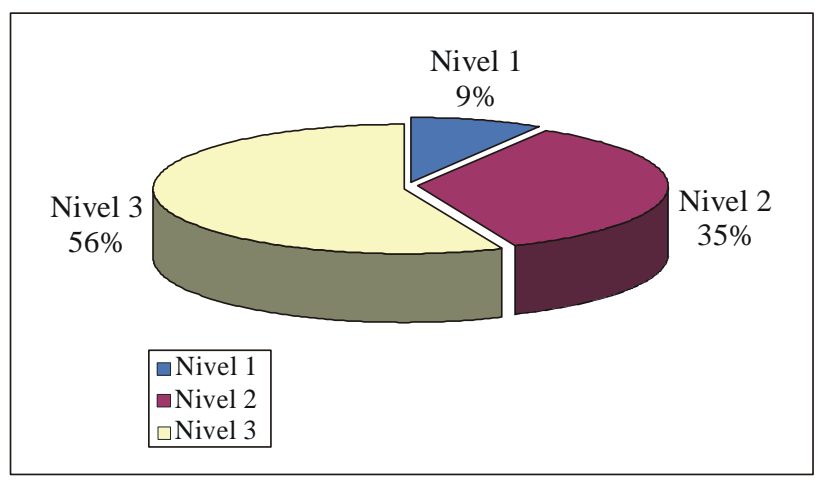

Figura 2.- Nivel de cumplimiento de normas de bioseguridad. Profesionales y técnicas de enfermería. HNAAA 2002. 
El grado de conocimiento acerca de normas de bioseguridad del personal profesional comparado con la técnica de enfermería se obtuvo que en el servicio de UCI y Centro quirúrgico; $100 \%$ de enfermeras y técnicos de enfermería tienen alto grado de conocimiento. En otros servicios es parejo en ambos estratos, excepto en hemodiálisis y UCEMIN, en que la diferencia es marcada (Tabla 4).

Tabla 4.- Grado de conocimiento de bioseguridad del personal enfermeras comparado con técnicos de enfermería.

\begin{tabular}{lccccc}
\hline \multirow{2}{*}{ Servicio } & \multicolumn{5}{c}{$\begin{array}{c}\text { Grado de conocimiento de } \\
\text { bioseguridad }\end{array}$} \\
\cline { 2 - 6 } & Enfermeras & \multicolumn{3}{c}{ Técnicas } \\
\cline { 2 - 6 } \cline { 5 - 6 } & $\begin{array}{c}\text { Alto Regular } \\
\%\end{array}$ & $\begin{array}{c}\text { Alto } \\
\%\end{array}$ & $\begin{array}{c}\text { Regular } \\
\%\end{array}$ & $\begin{array}{c}\text { Bajo } \\
\%\end{array}$ \\
\hline UCI & 100 & 0 & 100 & 0 & 0 \\
UCEMIN & 57 & 43 & 33 & 67 & 0 \\
Cirugía & 80 & 20 & 67 & 33 & 0 \\
C.Quirúrgico & 100 & 0 & 100 & 0 & 0 \\
Neonatología & 90 & 10 & 80 & 20 & 0 \\
Emergencia & 92 & 8 & 78 & 22 & 0 \\
Hemodiálisis & 83 & 17 & 0 & 50 & 50 \\
\hline
\end{tabular}

En el nivel de cumplimiento de las normas de bioseguridad por parte del personal de enfermería comparado con la técnica de enfermería, se obtuvo que tanto en el Centro quirúrgico como en Hemodiálisis las profesionales de enfermería tuvieron $100 \%$ de cumplimiento nivel 3 , mientras el personal técnico $100 \%$ cumplimiento nivel 3 en UCI; nivel bajo tuvieron enfermeras y técnicas en UCEMIN y cirugía (Tabla 5).

\section{DISCUSIÓN}

El nivel de conocimientos de las medidas de bioseguridad según las respuestas al cuestionario es alto en todos los servicios, siendo excelentes UCI y Centro Quirúrgico y el que tiene menor nivel es UCEMIN. Los resultados son similares a los encontrados en otros estudios, tanto nacionales como de otros países latinoamericanos $\left({ }^{9-12}\right)$, lo que indica que ya se ha logrado el nivel de información básico requerido, mejorando los datos obtenidos en el año 2000 en el mismo Hospital $\left({ }^{13}\right)$.

Es necesario destacar que la mayor parte del personal entrevistado presenta mayor conocimiento sobre técnicas de lavado/ secado de manos, tipos de jabones y uso de guantes. Sin embargo, a pesar de los resultados obtenidos sobre grados de conocimiento, los de cumplimiento fueron diferentes.

No obstante que la mayor parte de la población observada presentó buenos niveles de cumplimiento de las normas de Bioseguridad con 4 servicios nivel 3 y los otros 3 servicios nivel 2 , se evidenció algunos errores comunes en casi todos los servicios. Entre ellos destaca el mal uso de guantes, pues después de manipular fluidos orgánicos o realizar procedimientos, el personal permanece con ellos más tiempo del debido, no realizando cambio oportuno después de la atención de cada paciente, lo que se suma a una menor frecuencia de lavado de manos.

Otro aspecto importante es la falta de uso de lentes o máscaras protectoras en casi todo el personal observado, a pesar de que algunos servicios sí cuentan con dicho material. Asimismo, se encontró desconocimiento sobre la concentración de la lejía que el mismo personal utiliza y la práctica peligrosa de reencapuchar las agujas luego de su uso.

En lo referente al manejo de los residuos sólidos, es la clasificación de los mismos la que presenta mayor déficit. Se aduce que no todos los servicios disponen de envases adecuados a cada tipo de residuos. Aunque este déficit no es cuantitativo sino cualitativo, esto provoca que el personal utilice las bolsas de que dispone, aunque no sean los específicos para el tipo de residuo que se está desechando. En algunos de los servicios se halló bolsas negras; lo mismo sucede en los servicios higiénicos de algunas áreas. En Hemodiálisis, se encontró bolsas 
Tabla 5.- Nivel de cumplimiento de medidas de bioseguridad del enfermeras comparado con técnicas de enfermería.

\begin{tabular}{|c|c|c|c|c|c|c|}
\hline \multirow[t]{4}{*}{ Servicio } & \multicolumn{6}{|c|}{$\begin{array}{l}\text { Nivel de cumplimiento medidas } \\
\text { bioseguridad }\end{array}$} \\
\hline & \multicolumn{3}{|c|}{$\begin{array}{c}\text { Enfermeras } \\
\text { Niveles }\end{array}$} & \multicolumn{3}{|c|}{$\begin{array}{l}\text { Técnicas } \\
\text { Niveles }\end{array}$} \\
\hline & 1 & 2 & 3 & 1 & 2 & 3 \\
\hline & $\%$ & $\%$ & $\%$ & $\%$ & $\%$ & $\%$ \\
\hline UCI & 0 & 33 & 67 & 0 & 0 & 100 \\
\hline UCEMIN & 43 & 57 & 0 & 33 & 67 & 0 \\
\hline Cirugía & 20 & 80 & 0 & 33 & 67 & 0 \\
\hline C.Quirúrgico & 0 & 0 & 100 & 0 & 100 & 0 \\
\hline Neonatología & 0 & 5 & 95 & 0 & 10 & 90 \\
\hline Emergencia & 0 & 45 & 55 & 22 & 88 & 0 \\
\hline Hemodiálisis & 0 & 0 & 100 & 25 & 0 & 75 \\
\hline
\end{tabular}

amarillas usadas para colocar el calzado de los pacientes hemodializados. También se observó consumo de alimentos en varios de los servicios, por ejemplo en Centro quirúrgico; en el área semirígida existe un ambiente en el cual se consume alimentos.

Cuando se compara el grado de conocimientos entre el personal profesional con las técnicas de enfermería, se observa que existen algunos servicios en los cuales la diferencia entre ambos es notable, como Hemodiálisis, en que las enfermeras tienen $83 \%$ alto y $17 \%$ regular, mientras que sus técnicas ninguna posee conocimiento alto $0 \%$ y $50 \%$ es regular y bajo. Comparando el nivel de cumplimiento entre profesionales y técnicos, se aprecia que los servicios de Emergencia, Cirugía y Centro Quirúrgico cuentan con personal de enfermería y técnico que presenta diferentes niveles de cumplimiento.

Se observó que tanto enfermeras y técnicos en el servicio de Unidad de Cuidados Intensivos, si bien obtuvieron conocimiento alto en el $100 \%$, el cumplimiento en las enfermeras fue de $67 \%$ nivel 3 y $33 \%$ nivel 2 , en los técnicos se mantuvo en nivel de cumplimiento nivel 3 , mientras que el personal técnico y de enfermería de los servicios de Centro Quirúrgico y Neonatología tuvieron un grado de conocimiento alto sobre normas de bioseguridad, que coincidió con un nivel de cumplimiento elevado (nivel 3 ).

Se ha encontrado similitudes con los otros estudios latinoamericanos en los que, existiendo un buen nivel de conocimientos de bioseguridad, la aplicación o cumplimiento de las normas o uso de medidas de prevención no son en el grado que debería existir.

Por lo que, es importante lograr la concientización adecuada del personal que trabaja en áreas de alto riesgo del hospital sobre la importancia del cumplimiento de las normas de bioseguridad, pues si bien tienen un grado de conocimiento alto, contrasta mucho con el nivel de cumplimiento de las mismas y a pesar de ser conocido hay que insistir principalmente en: No consumir alimentos dentro de su centro de trabajo, en las áreas y servicios de alto riesgo, realizar una correcta clasificación de los residuos para su adecuado manejo en bolsas correspondientes y desechar agujas u otros objetos punzo-cortantes sin reencapuchar en recipientes cerrados, rígidos, rotulados y no perforables, pues de otro modo se tendría problemas serios en la salud de los trabajadores asistenciales.

\section{Agradecimiento}

A León Alcántara, Carla; Llontop Panta, Margarita, López Torres, Lissett; Lozano Burgos, Judit, y Miranda Alcántara, María L, estudiantes del sexto año de Medicina Humana de la Universidad Nacional Pedro Ruiz Gallo, por su colaboración en la realización de las encuestas.

\section{REFERENCIAS BIBLIOGRÁFICAS}

1. Essalud. Normas Generales de Bioseguridad en EsSalud. Centro de Prevención de riesgos de Trabajo. Lima: EsSalud; 2001.

2. Malagón-Londoño, Galán M, Pontón L. Administración Hospitalaria. Primera edición. Buenos Aires: Editorial Médica Panamericana; 1998. 
3. IPSS. Manual de Bioseguridad y Salud Ocupacional Hospitalaria. Programa Central de Servicios Especiales. Lima: IPSS; 1997.

4. Organización Mundial de la Salud (OMS/OPS). Manejo de Desechos Médicos en Países en desarrollo. Informe de consultoría. Ginebra: OMS; 1992.

5. Hospital Nacional Almanzor Aguinaga Asenjo. Procedimientos Generales y Codificación oficial para la recolección y disposición de los residuos sólidos hospitalarios. Curso-Taller de Bioseguridad. Centro de Prevención de Riesgos de Trabajo. Gerencia de Red Asistencial. Lambayeque: Hospital Nacional Almanzor Aguinaga Asenjo; 2001.

6. Cantanhede A. La Gestión y Tratamiento de los Residuos generados en los Centros de Atención de Salud. En: Encuentro de Especialistas en tratamiento y destino final de residuos del servicio de Saúde. Repertorio Científico 1999; 5(6-7).

7. Milliam D. Puesta al día sobre el control de las Infecciones. N Nursing 1994;12(5):17-20.

8. Verde J, Costabel M. Bioseguridad en Enfermería. Montevideo: Editorial San Martín; 1994.

9. Elguren M. Bioseguridad [en línea]. Buenos Aires: Monografias.com; 18 Dic. 2001. http://www.monografias.com [Consulta: 15 Junio 202]
10. Fernández FO, Bastías SJ, Venegas CC. Evaluación del conocimiento y cumplimiento de las normas de bioseguridad en la atención odontológica. Región Metropolitana (19951996). Rev Chil Salud Pública 1998;2(1):74-8.

11. Agudelo CR, Rendón OI, Palacio VJ. Gestión integral de residuos sólidos peligrosos y cumplimiento de normas de bioseguridad en laboratorios de tanatopraxia, Medellín 2001. Rev Fac Nac Salud Pública 2003;21(1):43-53.

12. Condori JP. Estudio Comparativo de Conocimientos y Actitudes relacionados al VIH / SIDA y sus normas de bioseguridad entre el personal médico y paramédico del Hospital Carlos Monge Medrano y el Hospital del IPSS Juliaca. [Tesis de Bachiller Medicina]. Arequipa: Universidad Nacional San Agustín; 1997.

13. Soto-Cáceres V, Olano D E. Conocimiento de las normas de bioseguridad por el personal asistencial del Hospital Nacional Almanzor Aguinaga Asenjo. Trabajo de Investigación Cátedra de Medicina Preventiva. Lambayeque: Facultad de Medicina Humana, Universidad Nacional Pedro Ruiz Gallo; 2001.

14. Oficina General de Epidemiología. Protocolo para el estudio de conocimientos, actitudes y prácticas del personal de salud en el control de las infecciones intrahospitalarias. OGERENACE/VIGIA. Lima: Gráfica Bellido; 2000. 